\title{
CARDIOEMBOLIC STROKE IN LATVIA: PREVENTION AND LONG-TERM OUTCOME
}

\section{Elina Pucite ${ }^{1}$, Kristaps Jurjāns ${ }^{2}$,Evija Miglāne ${ }^{3}$, Baiba Luriṇa ${ }^{4}$, Oskars Kalējs ${ }^{5}$, Andrejs Millers ${ }^{6}$, Zanda Priede ${ }^{7}$}

\begin{abstract}
INTRODUCTION: Untreated non-valvular atrial fibrillation is one of major causes of stroke. The goal of the study was to evaluate the use of antithrombotic medication stroke prevention and assess long-term stroke outcome.

METHODS: This study involved 531 cardio embolic stroke patients of the Paul's Stradins Clinical University Hospital, Riga, Latvia, in 2014. After discharge the patients or their relatives were interviewed by phone after $30,90,180$, and 365 days. Standardized questions were asked about the patients' abilities and use of prescribed secondary prevention medication. The results were compared between patient groups, assigned according to prescribed medications.

RESULTS: Of all the patients included in the study, $8.9 \%$ were using oral anticoagulants before stroke onset. One year after discharge, $1.44 \%$ of patients were not using any preventive medication, $23.56 \%$ were using antiplatelet agents, $43.27 \%$ warfarin, and $31.73 \%$ target-specific oral anticoagulants. The one-year mortality rate was $40.7 \%$. The mortality rate was significantly higher in the patient group using no secondary preventive medication or antiplatelet agents compared to the patient group that used oral anticoagulants.

CONCLUSION: Cardio embolic stroke primary and secondary prevention in Latvia is lacking. The study outcomes suggest that action is needed to increase the use of oral anticoagulants in primary stroke prevention in patients with atrial fibrillation. Poor function outcomes, dementia, and patients' incompliance limits the use of oral anticoagulants in secondary prevention.
\end{abstract}

UDC Classification: 616.8 DOI: http://dx.doi.org/10.12955/cbup.v4.821

Keywords: Atrial fibrillation, cardio embolic stroke, stroke mortality, primary stroke prevention, secondary stroke prevention.

\section{Introduction}

As previously noted in Jurjans, et al. (2015, p. 199), "Non-valvular atrial fibrillation (AF) is the most common form of heart arrhythmias". With prevalence of about $1-2 \%$ in the general population with tendency to have increased over the last 50 years. Jurjans, et al. (2015, p. 199) also wrote:

The prevalence tends to increase with aging from $0.5 \%$ in 40-50 year old patients till 5-17\% in 8085 year old patients (Camm et al., 2012; European Heart Rhythm et al., 2010; Go et al., 2001; Stewart, Hart, Hole, \& McMurray, 2001). The possibility of developing AF at the age of 55 in the European population is $23.8 \%$ for men and $22.2 \%$ for women (Heeringa et al., 2006). AF is associated with greater cardio embolic accident risk, such as cardio embolic stroke, left ventricle hypertrophy, chronic heart failure, which lead to lower quality of life and higher mortality rates (Kirchhof et al., 2007). The use of oral anticoagulants decreases the risk of thromboembolic

\footnotetext{
${ }^{1}$ Elina Pucite, Pauls Stradins Clinical University Hospital, Department of Neurology and Riga Stradins University, Department of Neurology and Neurosurgery, Riga, Latvia, elina.pucite@ rsu.lv, elina.pucite@gmail.com

${ }^{2}$ Kristaps Jurjans, Pauls Stradins Clinical University Hospital, Department of Neurology, Riga, Latvia, Kristaps.jurjans@gmail.com

${ }^{3}$ Evija Miglane, Pauls Stradins Clinical University Hospital, Department of Neurology and

Riga Stradins University, Department of Neurology and Neurosurgery, Riga, Latvia, evija.miglane@ @stradini.lv

${ }^{4}$ Baiba Lurina, Pauls Stradins Clinical University Hospital, Department of Cardiology, Riga, Latvia,

baiba.lurina@gmail.com

${ }^{5}$ Oskars Kalejs, Pauls Stradins Clinical University Hospital, Department of Cardiology and

Riga Stradins University, Department of Internal diseases, Riga, Latvia, okalejs@gmail.com

${ }^{6}$ Andrejs Millers Pauls Stradins Clinical University Hospital, Department of Neurology and

Riga Stradins University, Department of Neurology and Neurosurgery, Riga, Latvia, andrejs.millers.stradini.lv

${ }^{7}$ Zanda Priede, Pauls Stradins Clinical University Hospital, Department of Neurology and

Riga Stradins University, Department of Neurology and Neurosurgery, Riga, Latvia, zada.priede@stradini.lv
} 
accidents in patients with non-valvular AF. To achieve effective cardio embolic accident prophylaxis, oral anticoagulants should be used regularly for prolonged period of time, which for most patients has proven to be difficult, considering the specific use of medication and possible side effects. The available oral anticoagulants in Latvia vitamin K antagonists (VKA) warfarin and target specific oral anticoagulants (TSOACs) -- rivaroxaban, dabigatran and apixaban.

Cardio embolic stroke is one of most severe forms of cerebral infarction, with rapid onset, severe disability, high morbidity, and poor functional outcome compared with non-AF-related strokes. Jurjans, et al. (2015, p. 199) also stated: "Stroke etiology in patients with AF varies from 1\%-20\% with average $4.5 \%$ a year and mortality of $30 \%$. Of all deaths associated with cardio embolism, $80 \%$ are caused by cerebral infarction (Menke, Luthje, Kastrup, \& Larsen, 2010).”

The objective of this study was to evaluate the use of oral anticoagulants in primary and secondary stroke prevention in patients with non-valvular atrial fibrillation.

As mentioned in Jurjans, et al. (2015, p. 199) "In 2012, the European Society of Cardiology (ESC) published the guidelines, "2012 Focused Update of the ESC Guidelines for the Management of Atrial Fibrillation", for treatment of non-valvular atrial fibrillation, which recommend that patients with AF should be evaluated by $\mathrm{CHA}_{2} \mathrm{DS}_{2}$-VASc and HAS-BLED scores to evaluate stroke and major bleeding risks accordingly (Lane \& Lip, 2012)". As defined in the guidelines, all patients with $\mathrm{CHA}_{2} \mathrm{DS}_{2}-\mathrm{VASc}$ score of two or above should be given oral anticoagulants and the "HAS-BLED should not be used to decide whether to offer anticoagulation in someone with a $\mathrm{CHA}_{2} \mathrm{DS}_{2}-\mathrm{VASc}$ score of 2 or above" Jurjans, et al. (2015, p. 200). As for the male patients with $\mathrm{CHA}_{2} \mathrm{DS}_{2}-\mathrm{VASc}$ a score of one, the benefit of oral anticoagulants must be compared with possible bleeding risk as assessed by the HAS-BLED score, with oral anticoagulants prescribed where the stroke risk outweighs the risk of bleeding. Stroke and bleeding risks should be re-evaluated at least once a year, coinciding with change of age category new hypertension, and certain other categories(Camm et al., 2012; Hobbs et al., 2015).

\section{Materials and Methods}

In 2014, at the Clinic of Neurology, Paul's Stradins Clinical University Hospital, Riga, Latvia, 1186 ischemic stroke patients were treated. In $531(44.77 \%)$ cases, non-valvular atrial fibrillation was diagnosed. Patients with hemorrhagic stroke, subarachnoid hemorrhage, or valvular AF were excluded from the study. Thus, a total of 531 patients were included in the data analysis. The average age of studied patients was 76.64 (SD 8.6) years, with patient ages ranging from 37 to 99 years. Of these patients, $188(35.4 \%)$ were males and $343(64.6 \%)$ were females. Patients were evaluated using $\mathrm{CHA}_{2} \mathrm{DS}_{2}$-VASc and HAS-BLED scores before potential stroke onset and information about primary prevention medication was collected (Lane \& Lip, 2012).

A modified Rankin scale was used to assess patients functional outcome on discharge from hospital, and then 30, 90, 180, and 365 days after discharge (Sulter, Steen, \& De Keyser, 1999).

Data of stroke survivors that were discharged from hospital were divided into four groups depending on their antithrombotic medication used for secondary prevention, and the groups were then compared. To determine the therapeutic management for secondary stroke prevention, we analyzed prescriptions for oral anticoagulants within 365 days after discharge. Standardized questions were asked about patients' abilities and usage of prescribed medication.

Collected data were analyzed using Microsoft Excel and SPSS 10. Absolute and relative frequencies were calculated based on cross-tables. For testing normal distribution, a Kolmogorov-Smirnov test was applied.

The study was approved by the ethical board of Riga Stradins University, Riga, Latvia. A verbal consent to participate was obtained from the patients, or their relatives, by telephone.

\section{Results}

We analyzed CDA2DS2-VASc and HAS- BLED scores $(\mathrm{n}=531)$ before the potential onset of stroke using non-parametric tests. The mean calculated CDA2DS2-VASc was 4.1 (SD 1.9) and mean HASBLED was 3.2 (SD 1.2). 
Antithrombotics use in the primary stroke prevention was found to be inappropriate. Before the onset of stroke $284(53.5 \%)$ patients did not use any antithrombotic medication, $200(37.7 \%)$ used antiplatelet agents, $46(8.7 \%)$ warfarin, and $1(0.2 \%)$ TSOACs (Figure 1).

In-hospital mortality was $14.9 \%$, and $452(85.1 \%)$ patients were discharged from hospital. In 21 (4.65\%) patients no antithrombotic therapy was prescribed for secondary prevention due to contraindications, and 158 (34.95\%) patients were discharged with antiplatelet agents because of severe neurologic deficit, the patient's or their relative's noncompliance, and patient's increased age and hemorrhagic transformation of cerebral infarction. The remaining $173(63.2 \%)$ patients were discharged with oral anticoagulants, 133 (29.43\%) patients with VKA and 140 (30.97\%) with TSOACs.

A degree of modified Rankin scale (mRs) was calculated for each patient at the time of discharge from the hospital with results between 1 and 3 considered a satisfactory outcome. At time of discharge 256 (48.21\%) patients had satisfactory functional outcome (mRs 0-3) and 196 (36.91\%) had severe disability (mRs $4-5)$, while $79(14.88 \%)$ had previously died in hospital. Only stroke survivors who were contacted $(n=348)$ were included in the further study. At 30 days since discharge from hospital, $201(57.75 \%)$ patients had a satisfactory outcome, $85(24.43 \%)$ were severely disabled, and 62 (17.82\%) had since died. After 90 days, $203(58.33 \%)$ of patients had satisfactory outcome, 67 (19.25\%) had severe disability, and 78 (22.42\%) had since died. At 180 days, 202 (58.06\%) patients had a satisfactory outcome, $22(6.31 \%)$ were severely disabled, and $124(35.63 \%)$ patients had since died. Finally, after one year, $195(56.05 \%)$ patients rated as satisfactory outcome, $13(3.73 \%)$ patients were severely disabled, and $140(40.22 \%)$ had since died (Figure 2$)$.

Figure 1: The use of antithrombotic medication in patients for different phases in the study

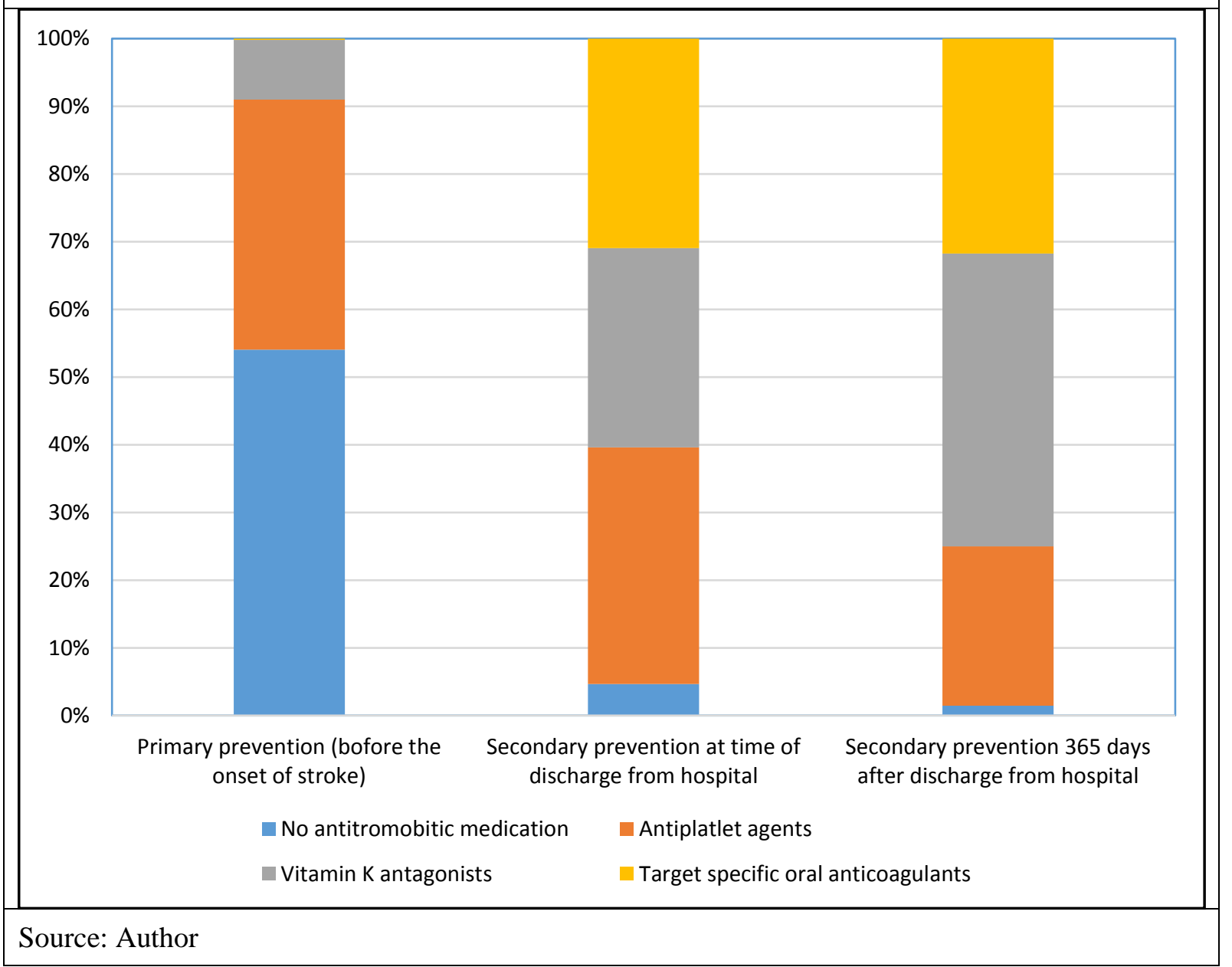


The 1-year mortality rate was as high as $40.7 \%$. The most common causes of death were somatic complications due to severe disability and recurrent cardiovascular accidents. The mortality rate 365 days after hospital discharge in patients using no secondary prevention antithrombotic medication $(70 \%)$ or antiplatelet agents $(71.31 \%)$ was significantly higher than in the patient groups using warfarin $(17.34 \%)$ or TSOACs $(19.44 \%)$ ( $\mathrm{p}<0.05)$.

On discharge, 179 (39.6\%) patients had no oral anticoagulants prescribed. Of these patients, only 52 (29.05\%) had a satisfactory stroke outcome (mRs 0-3) and 127 (70.95\%) patients were severely disabled (mRs 4-5).

Figure 2: Modified Rankin scale (mRs) at time of discharge from hospital and 30, 90, 180, and 365 days after discharge

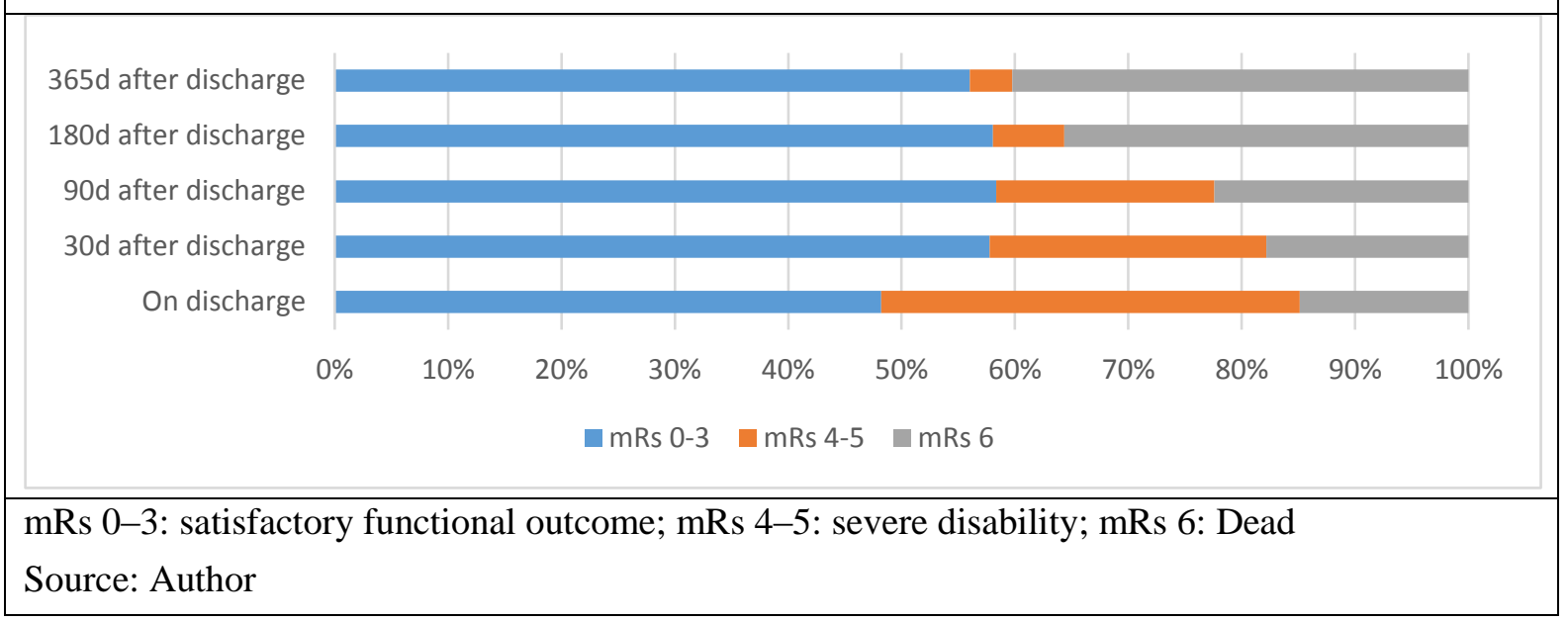

In our study, $208(39.17 \%)$ patients were aged 80 years or over. Of these patients, $33(15.86 \%)$ died in hospital. Of 137 patients that were contacted, 35 (22.54\%) had died in the 30 days after leaving hospital, $46(33.57 \%)$ in 90 days. In the 180 days after discharge the mortality rate was as high as 75 out of 137 patients (54.74\%) and one year after discharge this was 82 out of 137 (59.85\%). Both were considerably higher than the average 1-year mortality rate of $40.7 \%$.

Of 175 elderly patients discharged, $12(6.86 \%)$ had no preventive antithrombotic medication prescribed, while $90(51.43 \%)$ patients were prescribed antiplatelet agents, 34 (19.43\%) VKA, and 39 (22.28\%) TSOACs.

One year after discharge, a change of secondary prophylaxis was documented. In the stroke survivor group that did not start secondary prophylaxis $(\mathrm{n}=6)$, due to contraindications, two $(33.33 \%)$ had not started using any antithrombotic medication, two (33.33\%) had started using antiplatelet agents, one (16.67\%) VKA, and one (16.7\%) TSOACs. In the patient group discharged using antiplatelet agents $(\mathrm{n}=36)$, mainly because of a significant neurological deficit, one $(2.77 \%)$ had stopped using any antithrombotic medication, $21(58.34 \%)$ had continued with antiplatelet agents, and $11(30.5 \%)$ ambulatory patients had started using VKA, and three (8.34\%) had TSOACs. In the patient group discharged using VKA $(\mathrm{n}=80), 11(13.75 \%)$ had changed to antiplatelet agents for reasons unknown, $57(71.25 \%)$ had continued using warfarin, but $12(15 \%)$ had started using TSOACs. Patients that changed to TSOACs admitted that they experienced problems controlling their international normalized ratio (INR) relating to blood coagulation. In the patient group discharged using TSOACs $(\mathrm{n}=86), 15(17.44 \%)$ had changed to antiplatelet agents after consulting with a general practitioner, $50(58.14 \%)$ were still using TSOACs, and $21(24.41 \%)$ had changed to warfarin, mainly because of financial reasons (Figure 3).

Our study showed that, one year after discharge of 112 patients taking VKA, $83(74.1 \%)$ were therapeutically anticoagulated (INR $\geq 2.0), 18(16.1 \%)$ had sub-therapeutic INR $(<2.0)$, and $11(9.8 \%)$ showed no control with the INR. 


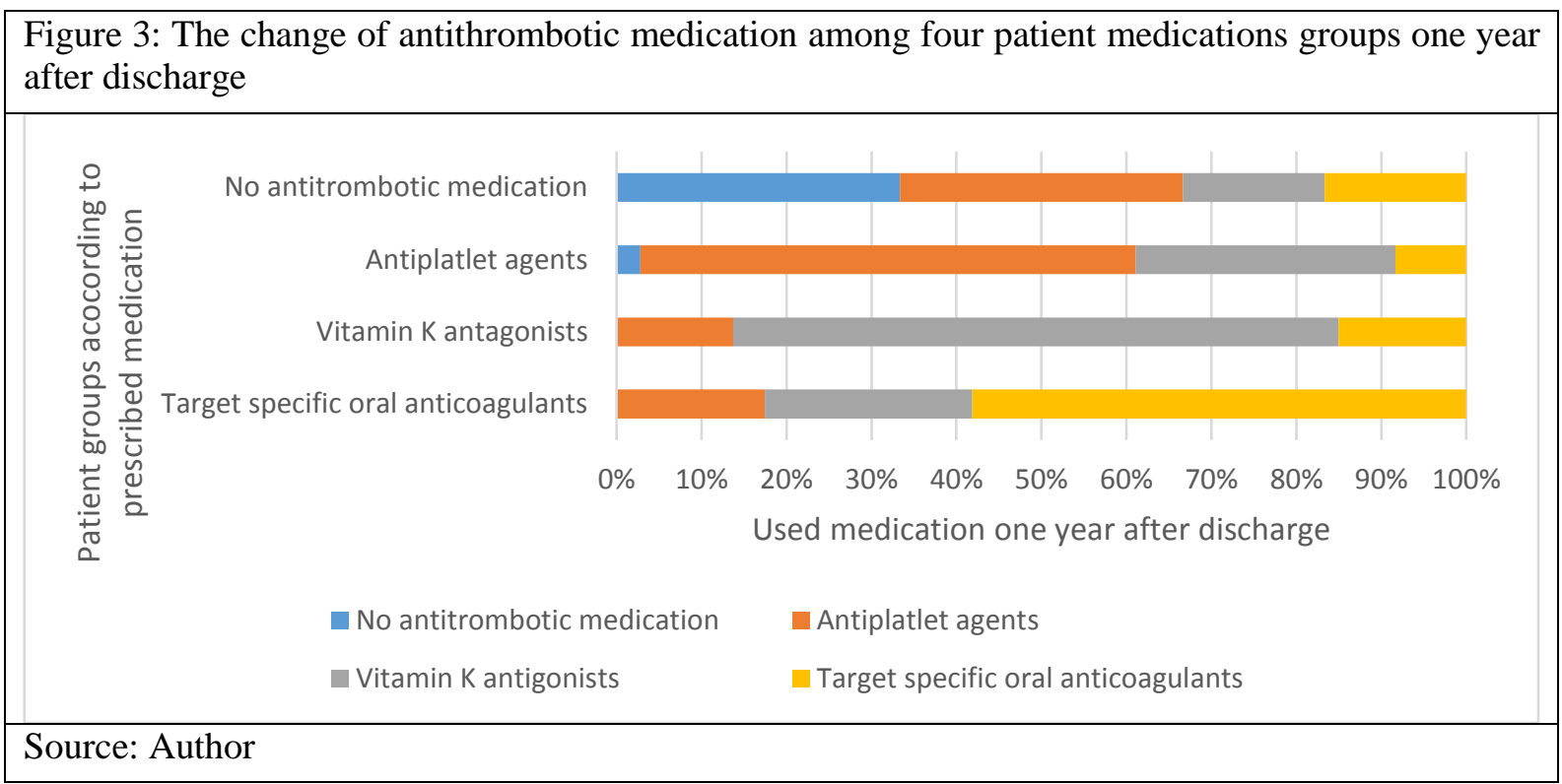

\section{Discussion}

As previously described in Jurjans, et al. (2015, p. 201), "Primary cardio embolic stroke prevention in Latvia is insufficient. According to ESC guidelines oral anticoagulants should be used if $\mathrm{CHA}_{2} \mathrm{DS}_{2}-$ VASc score is 1 for males and 2 for females", but $91.1 \%$ patients in our study, with average scores for $\mathrm{CHA}_{2} \mathrm{DS}_{2}$-VASc of 4.1 (SD 1.9) were using inadequate medication or were not using any preventive antithrombotic medication. Also, as noted in Jurjans, et al. (2015, pp. 202-3),

According to the European guidelines, women with a $\mathrm{CHA}_{2} \mathrm{DS}_{2}$-VASc score of 1 should not be given anticoagulation therapy on the basis of their sex alone as the risk of men and women with $\mathrm{CHA}_{2} \mathrm{DS}_{2}$-VASc scores of 1 is considered to be low and this group is not expected to benefit from anticoagulation therapy.

In a recent study of Friberg, Skeppholm, \& Terent (2015), patients with a $\mathrm{CHA}_{2} \mathrm{DS}_{2}-\mathrm{VASc}$ score of 1 for men showed no benefit from using oral anticoagulants as annual stroke risk ranged between $0.5 \%$ and $0.7 \%$. Overall, oral anticoagulant use was more common among low-risk patients than among patients with a $\mathrm{CHA}_{2} \mathrm{DS}_{2}$-VASc score $\geq 3$ (Friberg et al., 2015). Based on this article newly adapted US guidelines recommend treatment from score 2 and higher, with an option to treat cases with score 1 with oral anticoagulants, antiplatelet agents, or no medication (Friberg et al., 2015).

A Canadian study published in 2009 included 597 stroke sufferers with previously diagnosed atrial fibrillation (Gladstone et al. 2009). Of these patients, $40 \%$ were using anticoagulants, $30 \%$ were using antiplatelet agents, and $30 \%$ were not taking any antithrombotic medication before the onset of stroke. According to Gladstone, "Of those taking warfarin, three fourths had a sub-therapeutic international normalized ratio (INR) range $(<2.0)$ at the time of stroke admission. Overall, only $10 \%$ of patients with acute stroke with known atrial fibrillation were therapeutically anticoagulated (INR $\geq 2.0$ ) at admission" (Gladstone et al., 2009, p. 1). Jurjans et al. (2015, p. 202) wrote, "Our study did not specify if patient had previously diagnosed atrial fibrillation". Similarly, our results showed that $53.5 \%$ of patients did not use any antithrombotic medication, $37.7 \%$ of patients used antiplatelet agents, and many less used anticoagulants, with VKA in $8.7 \%$ of cases or TSOACs in $0.2 \%$ of cases. In both studies, treatment of more than $90 \%$ of patients were inadequately coagulated. Our study showed that 365 days after discharge from the neurologic department, $83(74.1 \%)$ patients using VKA were therapeutically anticoagulated (INR $\geq 2.0), 11(9.8 \%)$ could not control their INR, and 18 $(16.1 \%)$ had sub-therapeutic INR $(<2.0)$.

Of 452 patients discharged from the hospital, in 5.7\% antithrombotic medication was not recommended due to contraindications, $35.1 \%$ were discharged on antiplatelet agents, $28.2 \%$ on warfarin and $31 \%$ on TSOACs. 
As previously stated by Jurjans, et al. (2015) the most common causes for avoiding oral anticoagulants in as many as $31.1 \%$ of the cases were severe neurologic deficit, dementia, patients or their relative's noncompliance, increased age and hemorrhagic transformation of cerebral infarction but not because of patients age and gender as reported in a similar study published in Germany by Tanislav et al. in 2014. This study included 6261 patients after transient ischemic attack or ischemic stroke who had been diagnosed with atrial fibrillation. They reported that only $45 \%$ patients were prescribed oral anticoagulants after discharge. According to our data in oral anticoagulants were prescribed more frequently, in $59.2 \%$ of patients but it is also considered insufficient.

Although antiplatelet agents have been proven ineffective for stroke prevention in patients with atrial fibrillation, both studies showed that antiplatelet agents are prescribed far too often.

Jurjans, et al. (2015, p. 202) also wrote: "In November 2014, in United Kingdom, Moran, Calvert, Feltham, \& Marshall (2014)" published a project about "a retrospective case review of patients with first ever stroke or TIA, who had possibly preventable or curable disease before the onset."

Four categories of missed opportunities for stroke/TIA prevention will be sought: untreated high blood pressure in patients eligible for treatment (either blood pressure $\geq 160 / 100$ or $\geq 140 / 90 \mathrm{~mm} \mathrm{Hg}$ in patients at high cardiovascular disease (CVD) risk); patients with atrial fibrillation with high stroke risk and no anticoagulant therapy; no lipid modifying drug therapy prescribed in patients at high CVD risk or with familial hypercholesterolemia (Moran et al., 2014).

When this study will be completed and published, just as Jurjans, et al. (2015, p. 203) expressed, "there will be an adequate data about primary stroke prevention for patients with AF in UK so our data could be compared". We are especially interested in reasons as Jurjans, et al. (2015, p. 202) said "for not taking oral anticoagulants in patients with AF".

study published in 2010 the reported cardio embolic stroke in hospital mortality rate is as high as $27.3 \%$ compared to our study's $14.88 \%$ "Some studies suggest that usage of oral anticoagulants for elderly patients to prevent embolic accidents does not outweigh the risk for bleeding (Friberg et al., 2015)".

In December 2014, Lip et al., as mentioned by Jurjans et al. (2015, p. 203):

studied 4130 elderly patients in France that were using VKA and compared them to a group of patients that were no taking any antithrombotic therapy". Conclusion was that elderly patients with $\mathrm{AF}$ have a higher risk of stroke and bleeding, but the benefits of VKA therapy for stroke/thromboembolism or mortality were present regardless of increasing age (Lip et al., , 2014).

In our study $208(39.17 \%)$ patients were 80 or more years old. Of these patients $33(15.86 \%)$ died in hospital. Of 137 patients that were contacted 35 (22.54\%) had died in 30 days after leaving hospital, in 90 days $46(33.57 \%)$, in 180 days the mortality rate is as high as 75 out of $137(54.74 \%)$ and 82 out of $137(59.85 \%)$ one year after discharge, that is significantly higher than the average one-year mortality rate of $40.7 \%$.

Of 175 elderly patients discharged, $12(6.86 \%)$ had no preventive antithrombotic medication prescribed; antiplatelet agents were prescribed for 90 (51.43\%) patients, VKA for $34(19.43 \%)$, and TSOACs for $39(22.28 \%)$.

In our study, in-hospital mortality was $14.88 \%$, which was relatively high, but mortality in 90 days was $26.62 \%$, and $38.0 \%$ in 180 days after discharge is high. One year after discharge $40.7 \%$ mortality rate is even hard to imagine. As Jurjans, et al. (2015, p. 203) described,

High mortality is associated with severe disability due to stroke, poor compliance using prescribed medication and mistakes in national health care organization. In other European countries stroke in hospital mortality is about $12.1 \%$, the mortality rate in 30 days after onset in Switzerland was reported 13\% (Nedeltchev et al., 2010) and mortality in 90 days doesn't exceed 15.7\% (Ois et al., 2007).

A study published in 2010, reported cardio embolic stroke in hospital mortality rate as high as $27.3 \%$ compared to our study's $14.88 \%$ (Arboix \& Alio, 2010). 


\section{Conclusion}

In Latvia, primary and secondary prevention of cardio embolic stroke is insufficient and mortality at 90, 180, and 365 days after discharge is very high. As Jurjans, et al. (2015, p. 203) concluded, "It is essential to raise patient awareness about cardio embolic stroke and its prevention as well as ensure that physicians prescribe oral anticoagulants for patients with atrial fibrillation and high risk for cerebral infarction, according to published guidelines".

\section{References}

Arboix, A., \& Alio, J. (2010). Cardio embolic stroke: clinical features, specific cardiac disorders and prognosis. Curr Cardiol Rev, 6(3), 150-161. DOI:10.2174/157340310791658730

Camm, A. J., Lip, G. Y., De Caterina, R., Savelieva, I., Atar, D., Hohnloser, S. H., \& Guidelines, E. S. C. C. f. P. (2012). 2012 focused update of the ESC Guidelines for the management of atrial fibrillation: an update of the 2010 ESC Guidelines for the management of atrial fibrillation. Developed with the special contribution of the European Heart Rhythm Association. Eur Heart J, 33(21), 2719-2747. DOI:10.1093/eurheartj/ehs253

European Heart Rhythm, A., European Association for Cardio-Thoracic, S., Camm, A. J., Kirchhof, P., Lip, G. Y., Schotten, U., \& Rutten, F. H. (2010). Guidelines for the management of atrial fibrillation: the Task Force for the Management of Atrial Fibrillation of the European Society of Cardiology (ESC). Eur Heart J, 31(19), 2369-2429. DOI:10.1093/eurheartj/ehq278

Friberg, L., Skeppholm, M., \& Terent, A. (2015). Benefit of anticoagulation unlikely in patients with atrial fibrillation and a CHA2DS2-VASc score of 1. J Am Coll Cardiol, 65(3), 225-232. DOI:10.1016/j.jacc.2014.10.052

Gladstone, D. J., Bui, E., Fang, J., Laupacis, A., Lindsay, M. P., Tu, J. V., \& Kapral, M. K. (2009). Potentially preventable strokes in high-risk patients with atrial fibrillation who are not adequately anticoagulated. Stroke, 40(1), 235-240. DOI:10.1161/STROKEAHA.108.516344

Go, A. S., Hylek, E. M., Phillips, K. A., Chang, Y., Henault, L. E., Selby, J. V., \& Singer, D. E. (2001). Prevalence of diagnosed atrial fibrillation in adults: national implications for rhythm management and stroke prevention: the AnTicoagulation and Risk Factors in Atrial Fibrillation (ATRIA) Study. JAMA, 285(18), 2370-2375.

Heeringa, J., van der Kuip, D. A., Hofman, A., Kors, J. A., van Herpen, G., Stricker, B. H., \& Witteman, J. C. (2006). Prevalence, incidence and lifetime risk of atrial fibrillation: the Rotterdam study. Eur Heart J, 27(8), 949-953. DOI:10.1093/eurheartj/ehi825

Hobbs, F. R., Taylor, C. J., Jan Geersing, G., Rutten, F. H., Brouwer, J. R., \& on behalf of the European Primary Care Cardiovascular Society, S. w. g. (2015). European Primary Care Cardiovascular Society (EPCCS) consensus guidance on stroke prevention in atrial fibrillation (SPAF) in primary care. Eur J Prev Cardiol. DOI:10.1177/2047487315571890

Kirchhof, P., Auricchio, A., Bax, J., Crijns, H., Camm, J., Diener, H. C., \& Breithardt, G. (2007). Outcome parameters for trials in atrial fibrillation: executive summary. Eur Heart J, 28(22), 2803-2817. DOI:10.1093/eurheartj/ehm358

Jurjâns, K., Sabeïnikova, S., Miglâne, E., Luriòa1, B., Kalçjs, O., Millers, A., \& Priede, Z. (2015). Problems of cardio embolic stroke primary and secondary prevention in the Latvian population. Proceedings of the Latvian Academy of Sciences. Section B, Vol. 69 (2015), No. 5(698), 199-204. DOI: 10.1515/prolas-2015-0029. Retrieved from https://www.degruyter.com/downloadpdf/j/prolas.2015.69.issue-5/prolas-2015-0029/prolas-2015-0029.xml

Lane, D. A., \& Lip, G. Y. (2012). Use of the CHA(2)DS(2)-VASc and HAS-BLED scores to aid decision making for thromboprophylaxis in nonvalvular atrial fibrillation. Circulation, 126(7), 860-865.

DOI:10.1161/CIRCULATIONAHA.111.060061

Lip, G. Y., Clementy, N., Pericart, L., Banerjee, A., \& Fauchier, L. (2014). Stroke and major bleeding risk in elderly patients aged $>/=75$ years with atrial fibrillation: the Loire Valley atrial fibrillation project. Stroke, 46(1), 143-150. DOI:10.1161/STROKEAHA.114.007199

Menke, J., Luthje, L., Kastrup, A., \& Larsen, J. (2010). Thromboembolism in atrial fibrillation. Am J Cardiol, 105(4), 502510. DOI:10.1016/j.amjcard.2009.10.018

Moran, G. M., Calvert, M., Feltham, M. G., \& Marshall, T. (2014). Retrospective case review of missed opportunities for primary prevention of stroke and TIA in primary care: protocol paper. BMJ Open, 4(11), e006622. DOI:10.1136/bmjopen2014-006622

Nedeltchev, K., Renz, N., Karameshev, A., Haefeli, T., Brekenfeld, C., Meier, N., \& Mattle, H. P. (2010). Predictors of early mortality after acute ischaemic stroke. Swiss Med Wkly, 140(17-18), 254-259. DOI:smw-12919

Stewart, S., Hart, C. L., Hole, D. J., \& McMurray, J. J. (2001). Population prevalence, incidence, and predictors of atrial fibrillation in the Renfrew/Paisley study. Heart, 86(5), 516-521.

Sulter, G., Steen, C., \& De Keyser, J. (1999). Use of the Barthel index and modified Rankin scale in acute stroke trials. Stroke, 30(8), 1538-1541.

Tanislav, C., Milde, S., Schwartzkopff, S., Sieweke, N., Kramer, H. H., Juenemann, M., \& Kaps, M. (2014). Secondary stroke prevention in atrial fibrillation: a challenge in the clinical practice. BMC Neurol, 14, 195. DOI:10.1186/s12883-0140195-y 